\title{
Standardization developments for large scale biobanks in smoking related diseases - a model system for blood sample processing and storage
}

\author{
Johan Malm¹, Thomas E Fehniger², Pia Danmyr ${ }^{3}$, Ákos Végvári ${ }^{2}$ Charlotte Welinder ${ }^{4}$, Henrik Lindberg ${ }^{5}$, \\ Paul Upton ${ }^{6}$, Stephanie Carter ${ }^{7}$, Roger Appelqvist ${ }^{2}$, Karin Sjödin ${ }^{3}$, Elisabet Wieslander ${ }^{4}$, Magnus Dahlbäck ${ }^{8}$, \\ Melinda Rezeli², David Erlinge ${ }^{9}$ and György Marko-Varga ${ }^{2,10^{*}}$
}

\begin{abstract}
Background: Biobank samples stored in biobanks give researchers and respiratory healthcare institutions access to datasets of analytes valuable for both diagnostic and research practices. The usefulness of these samples in clinical decision-making is highly dependent on their quality and integrity. New procedures that better preserve sample integrity and reduce degradation are being developed to meet the needs of both present and future biobanking. Hereby we present an automatic sample workflow scheme that is designed to handle high numbers of blood samples.

Methods: Blood fractions are aliquoted, heat sealed using novel technology, and stored in 384 tube high-density sample arrays.

Results: The newly developed 384 biobank rack system is especially suited for preserving identical small aliquots. We provide data on robotic processing of clinical samples at $-80^{\circ} \mathrm{C}$, following initial processing, analysis and shipping between laboratories throughout Europe. Subsequent to unpacking, re-sorting, and storage at these sites, the samples have been returned for analysis. Biomarker analysis of 13 common tests in the clinical chemistry unit of the hospital provides evidence of qualitative and stable logistics using the 384-sample tube system.
\end{abstract}

Conclusions: This technology development allows rapid access to a given sample in the frozen archive while maintaining individual sample integrity with sample tube confinement and quality management.

Keywords: Biobank; Biomarkers; Standardization; Robotics; Respiratory diseases

\section{Background}

The future of biomedical sciences will be driven by the ability to adopt novel technologies, generating large data sets of data, to understand disease and to develop new treatments. This is especially relevant to diseases such as lung cancer (LC) and chronic obstructive pulmonary diseases (COPD), responsible for high mortality and cost to the European health care system. Cancer, and especially lung cancer, as well as chronic obstructive pulmonary

\footnotetext{
* Correspondence: gyorgy.marko-varga@elmat.lth.se

${ }^{2}$ Clinical Protein Science \& Imaging, Biomedical Center, Dept. of

Measurement Technology and Industrial Electrical Engineering, Lund

University, BMC C13, 22184 Lund, Sweden

${ }^{10}$ First Department of Surgery, Tokyo Medical University, 6-7-1 Nishishinjiku

Shinjiku-ku, Tokyo 160-0023 Japan

Full list of author information is available at the end of the article
}

disease (COPD) are leading causes of smoking related mortality worldwide. Overall, a therapeutic plateau has been reached with conventional anti-cancer treatment strategies such as cytotoxic agents, radiotherapy and surgery. Given the still dismal survival rates, attention in recent years has focused on novel molecular targeted therapies with different mechanisms of action and better toxicity profiles. Accordingly, there is a clinical need to: (i) identify biomarkers that can help recognize patients that respond to these therapies, (ii) detect tumor resistance, (iii) and predict the efficacy of targeted drugs costeffectively. COPD is a progressive debilitating disease that often is not diagnosed before extensive tissue destruction has occurred. Prevalence rates of COPD vary across EU, ranging from 4 to $10 \%$ with an overall rate of 
respiratory symptoms between $45-65 \%$ across EU. Currently COPD is under-diagnosed and is associated with co-morbidity and risk for that increase the burden on the healthcare system, hospitalization costs and mortality. By implementing early detection, personalized treatment and evaluation of response to treatment both Lung Cancer and COPD have led to improved prognosis and reduced cost to the healthcare system. These smoking related respiratory diseases are multifactorial. Meaning that not a single molecule is related to one disease or clinical complaint, but rather to hundreds of molecules that are interconnected or pathway related in addition to protein complex formed. There is therefore a need for selecting from multiple read-out signals. However, this is hampered by the lack of tools and data for early diagnosis. One new direction that is proving to be a winning concept is the modeling of disease progression and evaluation of treatment responses.

Biobanking is gaining momentum with an increasing number of valuable patient samples linked with clinical information that are being stored in biobanks around the word [1,2]. The advancement in clinical research where high quality samples are utilized in research has increased significantly in the recent decade, but especially in the last years [3-10]. The targeted treatments with Personalized Medicines are becoming the new generation of drugs with high specificity and safety. In the developments of these new drugs, high quality patient samples are mandatory, where standardized Biobank resources will play a major role $[4,11]$. It has resulted in interest to share data, facilitated by biorepositories of specimens that is linked to health care information, where a lot of effort is given to meet the governance challenges of Biobank archives now and in the future [12]. Sample integrity is a real challenge, but also a mandatory requirement for any patient read out. In addition, life science clinical research material needs to use standard procedures, optimized protocols and processing cycle times, which are key aspects of work flows to consider $[13,14]$. Protein and molecular degradation is a well-known phenomenon that is directly related to sample handling procedures, where sample temperature, preparation times and sample preparation procedures are some variables that will be directly related to sample stability $[15,16]$. This is especially important when samples are stored frozen in 5-12 ml tubes, thawed and collected for analysis and then re-frozen again for future use. When this procedure is repeated multiple times, the composition of the original sample state is irreversibly changed. This gives rise to changes in absolute amounts of analytes that is not related to, e.g., the disease state of the patient sample. Processing times of blood might vary in-between some hours to 24 and 36 hours. The read-outs from samples that have been exposed to these extended processing times will vary. This is particularly critical to RNA analysis, metabolomics quantitation as well as protein expression analysis.

The processing protocols were worked out and reported in large-scale studies, such as the UK Biobank (http://www.ukbiobank.ac.uk/) and LifeGene (https://www. lifegene.se/). Keeping an absolute control of patient sampling, standardized procedures, validated sample handling protocols and electronic surveillance of the sample life cycle ranging from the needle in the arm of the patient to the automated storage handling at $-80^{\circ} \mathrm{C}$ will provide the basis for high quality biobanks $[2,14,17]$. New technology is changing a lot of the way that standard procedure in hospitals is performed. Sample degradation and sample losses due to evaporation along with cot effective sample archiving are among the long-standing issues that are most important in order to keep high quality of samples for long periods.

By the implementation of e-health logistics, efficient data storage and use, allows a data history to be established where the patient treatments are linked to the decision making of the physician, providing healthcare improvements [18]. A novel technology that relate to biomarkers and clinical status of the patients, is a great resource that utilizes blood samples as a major biofluid resource. Blood sample storages nowadays range from large national efforts into smaller development labs where the biospecimen collections are used as biobank assets, searching for healthcare solutions $[19,20]$.

The value that large scale biobanks provide paves the way for new areas that opens up the opportunities such as drug discovery, stem cell research and genetic research [21-23]. This study presents novel developments that improve the existing biobanking systems.

\section{Methods}

\section{Materials and instruments}

Matrix storage tubes and seals, $0.1 \mathrm{~mL} 384$ 2D tubes (Thermo Scientific 384 well 2D coded storage tubes part 3815, MA, USA). In the sealing experiments we used a WellMate dispenser (Thermo Scientific, MA, USA), a small bore tubing cartridge (Thermo Scientific, MA, USA). A 384 rack heat sealer (ALPS3000 Thermo Scientific, MA, USA), the Heat $20 \mu \mathrm{m}$ sealing foil (Easypierce, Thermo Scientific, MA, USA), the PTFE Type K couples TM Electronics (RS 409-4908, Thermo Scientific, MA, USA), 8 channel USB data logger, and Picotech picolog software (Pico technologies USBTC08). A Hamilton STAR Liquid Handling Platform (Hamilton, Reno, NV) was employed for automated aliquoting of blood samples. CO-RE 480 standard volume tips $(300 \mu \mathrm{L})$ with filters and CO-RE 480 standard volume tips $(1000 \mu \mathrm{L})$ without filters were purchased from Hamilton (Bonaduz, Switzerland). The variable temperature heat 
sealer instrument ALPS $^{\mathrm{TM}} 50 \mathrm{~V}$, Thermo Scientific, MA) was used for sealing 384-tubes with Easy Pierce $20 \mu \mathrm{m}$ heat-sealing tape (AB-1720, Lot No: 115895) (Thermo Fisher Scientific, MA). For registration of aliquots a VisionMate ${ }^{\circledR}$ scanner was used (Thermo Fisher Scientific, MA). Nautilus LIMS (Thermo Fisher Scientific, MA) was used throughout the entire study.

\section{Blood samples}

Blood samples were provided by healthy volunteers at the Skåne University Hospital, Malmö, Sweden. Samples were collected in the morning after breakfast intake. 10 $\mathrm{mL}$ blood was sampled repeatedly in primary tubes. EDTA sample types were centrifuged for 10 minutes at $10,000 \mathrm{rpm}$, which is the standard procedure at the hospital. $70-\mu \mathrm{L}$ aliquots were dispensed into 384-rack tubes (volume of $100 \mu \mathrm{L}$ ). These samples tubes were stored at $-80^{\circ} \mathrm{C}$ throughout the entire study, and on dry ice upon transportation.

The collection of blood samples was approved by the ethical board at Lund University (approval number: LU 532-03).

\section{Biomarker analysis}

All analyses were performed by experienced clinical chemistry staff at the Department of Clinical Chemistry, Skåne University Hospital, Malmö, Sweden. The laboratory is accredited by SWEDAC (Swedish Board for Accreditation and Conformity Assessment). The following tests were run using standard methods from Roche: $\alpha_{1}$-antitrypsin, ALAT (alanine aminotransferase), albumin, apoA (apolipoprotein A1), creatinine, CRP, cystatin C, estradiol, Fe, ferritin, bile acid, glucose, fibrinogen, haptoglobin, HCG, IgA, IgG, IgM, K, Mg, Na, EPK, LPK, TPK, TSH, total protein. All samples were analyzed on a Cobas 8000 modular analyzer from Roche (Basel, Switzerland).

\section{Results and discussion}

As the sample volume requirements are steadily decreasing within the clinical chemistry departments in hospitals around the world, blood sample handling from patients becomes a critical part in the healthcare logistics. We recently presented a 384 high density solution [17] that is currently being implemented as an efficient and cheap solution to Biobank archiving in clinical hospitals. The 384-rack is confined with a hard polymeric frame where the tubes (384) are mounted. Manual or robotic picking and sorting can be made for all of the tubes within the 384 biobank sample plate (10). The plates and foils have been pre-tested for the used conditions to make sure that there is no release of, e.g., plastifiers to contaminate the samples.

Here we provide standardization and stability data on the development of 384 Biobank sample systems, including an entire cycle of blood plasma samples that were processed and sealed in the hospital in Malmö, Sweden. The samples were stored at $-80^{\circ} \mathrm{C}$, transported throughout Europe to Lichtenstein, processed by $-80^{\circ} \mathrm{C}$ robotics and shipped back to the hospital in Malmö, as outlined in Figure 1.

\section{Sample tube confinement and quality management}

In order to be efficient and reliable, biobanks must adopt and implement best practices with acceptable and standardized sample tube processing for Biobank storage.

A 384 Sealer-Cutter (Thermo Fisher Scientific) was evaluated, providing experimental evidence on sample temperature maintenance throughout the 384-rack sealing process utilizing sealing temperatures $>150^{\circ} \mathrm{C}$. One important question in these optimizations was: Are foil seal tubes stable at low temperatures?

We ran the experiments at room temp, and storage after liquid nitrogen storage over a period of 30 days, and with a sample number of $n=30$ or 16 for each measured points. By positioning probes in the tubes, the probes were placed in the tube just below the meniscus of the liquid. The probes were inserted from the side to reduce the displacement of sample in the well. The probe wire was routed out of the rack down an adjacent tube. The base and one side of the tube are removed to allow the wire to pass through. The rest of the tube is kept where possible in tact to keep the thermal mass of the test rack as close to a standard unmodified rack as possible. Probe wires must be routed between tubes or around the skirt of the rack to allow the rack to sit on the base of the tubes during the sealing process. Holes in one end of the rack skirt allow the wires to exit the side of the rack without interfering with the sealing process.

We performed a tube preparation i) by removing two adjacent tubes from the rack; then ii) drilling a $1-\mathrm{mm}$ hole in one just above the ridge in the side. Next we removed the side and base of the other tube to the same height leaving the top of the tube intact. The probe wiring will exit through the cut away tube as shown in upper and lower images of Figure 2.

In order to investigate the temperature effect on the liquid within the 384 tube sets, the datalogger was set to log single measurements from the eight probes at 200 $\mathrm{ms}$ intervals and the log started prior to filling the racks. Next, tube racks were filled with $70 \mu \mathrm{L}$ of water. For the wells containing temperature probes the liquid may bridge across the well above the probe. It is necessary to use a disposable tip to break the surface tension below the probe so the liquid drops to the bottom of the tube. The filled rack was then transferred to the ALPS 3000 and heat sealed, while sealing the door of the instrument was held open to allow the wires to prevent the wires getting trapped. Two test runs were made sealing at $165^{\circ} \mathrm{C}$ for 1 second and then for 2 seconds. The same rack was 




Figure 1 Schematic illustration of the blood sample stability procedure conducted in order to investigate biomarker stability.

used with the foil removed for the second run. Further testing suggests sealing at $155^{\circ} \mathrm{C}$ for 0.5 seconds provides adequate sealing without deforming the tubes unduly. Sealing at 165 for 1 second melted more of the tube than is desirable. Fine-tuning and adjustments can be made at site and optimized accordingly.

The sample heating effects were investigated and presented in Figure 3A and 3B with 1 and 2 seconds of contact time at $165^{\circ} \mathrm{C}$. The heat transfer effects will be minimal with above conditions. Time will be sufficient for the heat to diffuse from the foil to the sample. This result in that the system is efficiently cooled before much of the heat is transfers to the sample.

Figure 3 shows the temperature profiles upon sealing. It can be seen from Figure 3B, that the temp rises to about $32^{\circ} \mathrm{C}$, and by lowering the time to 1 second it reaches about $28^{\circ} \mathrm{C}$. This small elevation of temperature will quickly return to ambient temperature as shown in Figure 3.

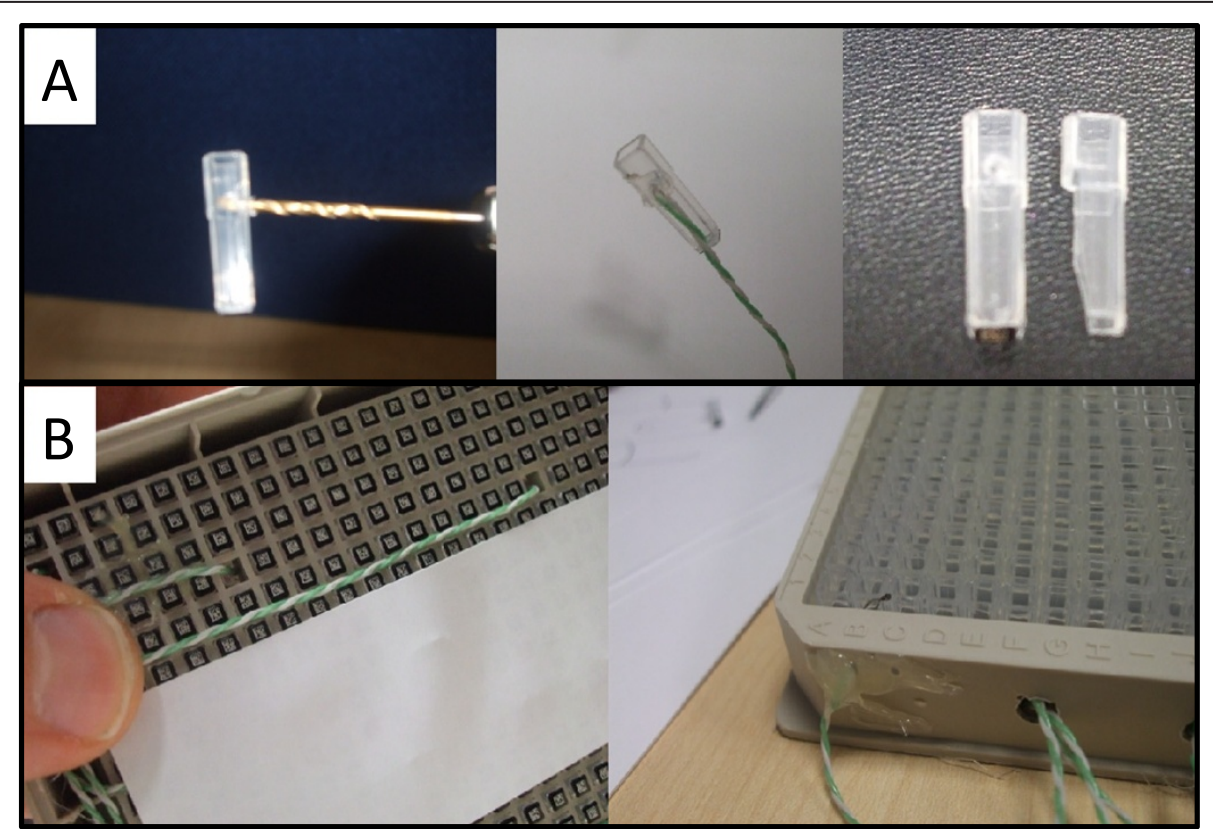

Figure 2 Photos showing the tube preparation procedures for the temperature stability experiments (A) and photos showing the mounting of the temperature control of the 384-tube system (B). 

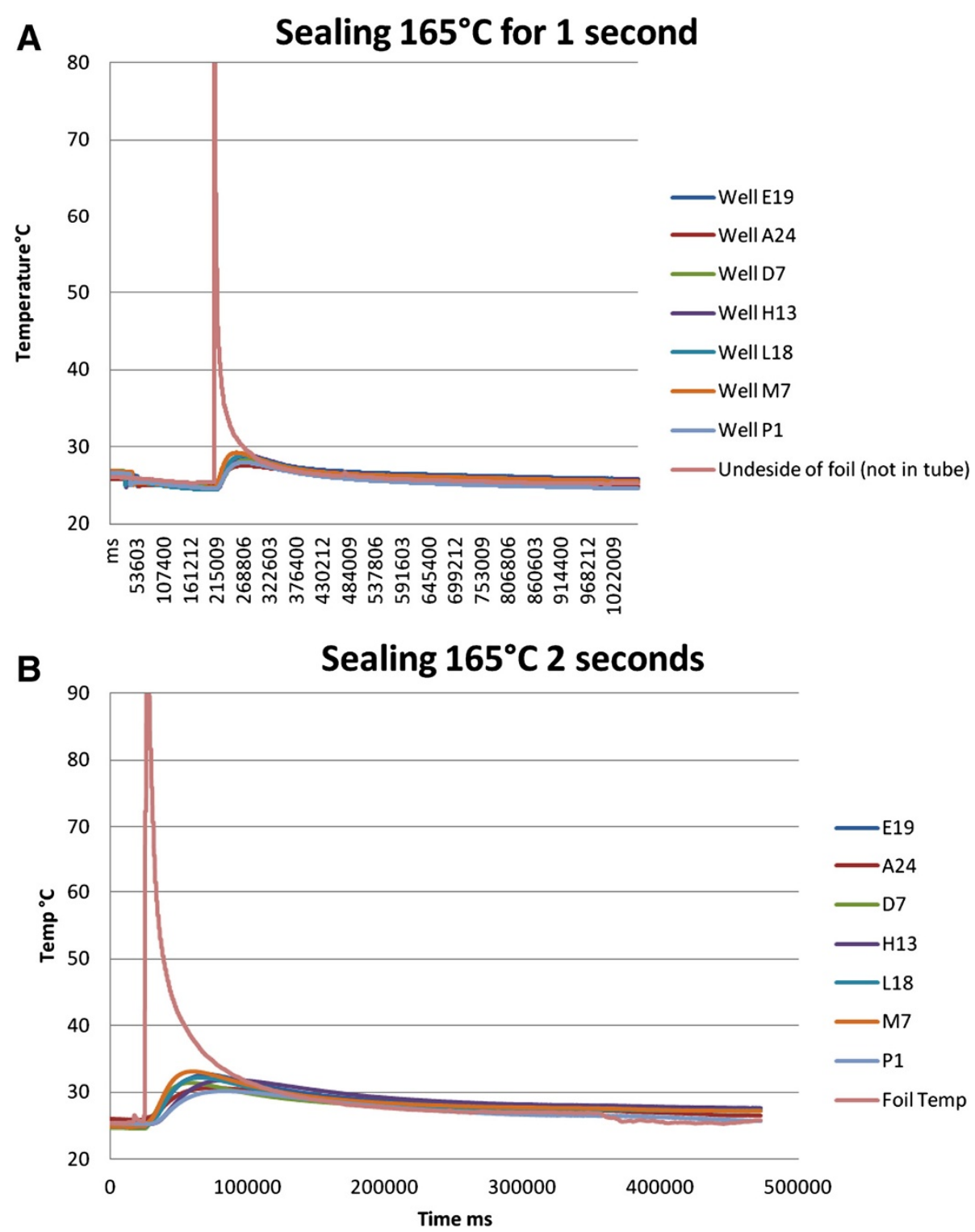

Figure 3 Temperature dependence of automated sealing of the 384 sample tube systems at $165^{\circ} \mathrm{C}$ for (A) 1 and (B) $2 \mathrm{~s}$. The different colors correspond to wells located in different regions of the rack.

The overall temperature dependencies are shown in Table 1 and these results indicate that the recommended sealing conditions for ALPS3000, $155^{\circ} \mathrm{C}$ for 0.5 seconds.

\section{Long term blood sample stability}

Long term stability of samples with respect to samples volumes and evaporation in any storage temperature is of major importance. Simply because a decreased volume and alteration in sample volumes in Biobank tubes will result in false diagnosis quantitations.

In order to investigate the tightness of our newly developed sealing, and the automated processing of 384 systems, we compared the weight of empty tubes, sealed empty tubes and tubes sealed with water inside.
By comparing the data at ambient temperatures the stability was investigated over a 30 day time period. We found in the case of the sealed tubes both empty or filled the results were found to be highly similar, providing evidence that the evaporation from the 384 high-density biobank tube system insulates well after

Table 1 Sealing temperature dependence

\begin{tabular}{lllll}
\hline $\begin{array}{l}\text { Sealing } \\
\text { condition }\end{array}$ & $\begin{array}{l}\text { Maximum } \\
\text { average } \\
\text { increase }\end{array}$ & $\begin{array}{l}\text { Time } \\
\text { to max } \\
\text { average }\end{array}$ & $\begin{array}{l}\text { Absolute max } \\
\text { increase in } \\
\text { individual well }\end{array}$ & $\begin{array}{l}\text { Time to max } \\
\text { in individual } \\
\text { well }\end{array}$ \\
\hline $165^{\circ} \mathrm{C} / 1$ second & $3.79^{\circ} \mathrm{C}$ & $59 \mathrm{~s}$ & $4.53^{\circ} \mathrm{C}$ & $45 \mathrm{~s}$ \\
$165^{\circ} \mathrm{C} / 2$ second & $6.47^{\circ} \mathrm{C}$ & $52 \mathrm{~s}$ & $8.39^{\circ} \mathrm{C}$ & $34 \mathrm{~s}$ \\
\hline
\end{tabular}






the sealing process (Figure 4). When calculating accelerated time frames in order to be able to extrapolate long-term stability, we have utilized the medical packaging accelerated ageing calculators (http://lso-inc.com/medicalpackage-testing/accelerated-aging.html). By using this calculation with the lower value (Q10), suggests that 1 day at $20^{\circ} \mathrm{C}$ is approximately equivalent to 1 year at $-80^{\circ} \mathrm{C}$.

\section{Robotic sample processing}

The automated handling of samples in the biobank freezers are in most cases separated from the actual archiving of the sample racks. Robotic sorting and picking of sample tubes from the sample rack by most instruments are performed at $-20^{\circ} \mathrm{C}$. However, newly introduced robotic developments have proven to manage automated operations at $-80^{\circ} \mathrm{C}$. This is of great value, since the both the storage and the sample tube handling is performed at the same temperature $\left(-80^{\circ} \mathrm{C}\right)$, where the samples are more stable.

Another sample stability consideration is the general principle that biobank samples are never re-used, i.e., that the samples from biobanks are never re-entered back to $-80^{\circ} \mathrm{C}$ storage. The reason for this strategy is to maintain the sample quality over time, which is impossible if the sample goes through repetitive freezethaw cycles. By utilizing high density 384-tube formats, with $70 \mu \mathrm{L}$ sample volumes, the single use approach can be fulfilled [17]. In order to maintain the sample integrity and quality over time, the principle of single usage is also gaining acceptance.

\section{Ultra low temperature robotic and 384-tube processing}

The sample integrity in clinical samples has to be maintained over longer time periods and provide a quality that can be used in global studies. The 384 high-density tube format provides this opportunity, and in order to be able to show the $-80^{\circ} \mathrm{C}$ robotic processing procedures a pilot experiment was conducted. Plasma samples $(n=768)$ in Sweden with racks of 384 tubes with a sample volume of $70 \mu \mathrm{L}$ were shipped to Lichtenstein on dry ice. 13 biomarkers were quantified within the plasma samples in Sweden as a baseline. The same samples shipped to Lichtenstein, processed by the $-80^{\circ} \mathrm{C}$ robotic picker and sorter (Kiwi, Liconic, Lichtenstein) in 10 cycles with a random sorting procedure. The robotic picker was operating constantly sorting the tubes in a random walk procedure within the 384-rack, from one position to the next. At no instance did the robotic arm drop or misplace the plasma sample tubes.

Next, the 384 plasma tube samples $(n=45)$ were shipped back to the University hospital in Malmö on dry ice and analyzed using the 13 biomarker assays. These assays were chosen by the standards of highest frequency tests in the hospitals in southern Sweden where the patients with various diseases are screened. As shown in Figure 5, the difference in-between the two analysis are negligible, which points to the fact that the $-80^{\circ} \mathrm{C}$ robotic processing does not influence the stability of blood plasma samples.

\section{Conclusions}

The current manuscript is of major relevance to the diagnostic respiratory field as it outlines the standardization and qualitative aspects of blood sample processing in large-scale biobanking.

Biobanks are dependent upon effective sample processing and stable long-term low temperature storage 


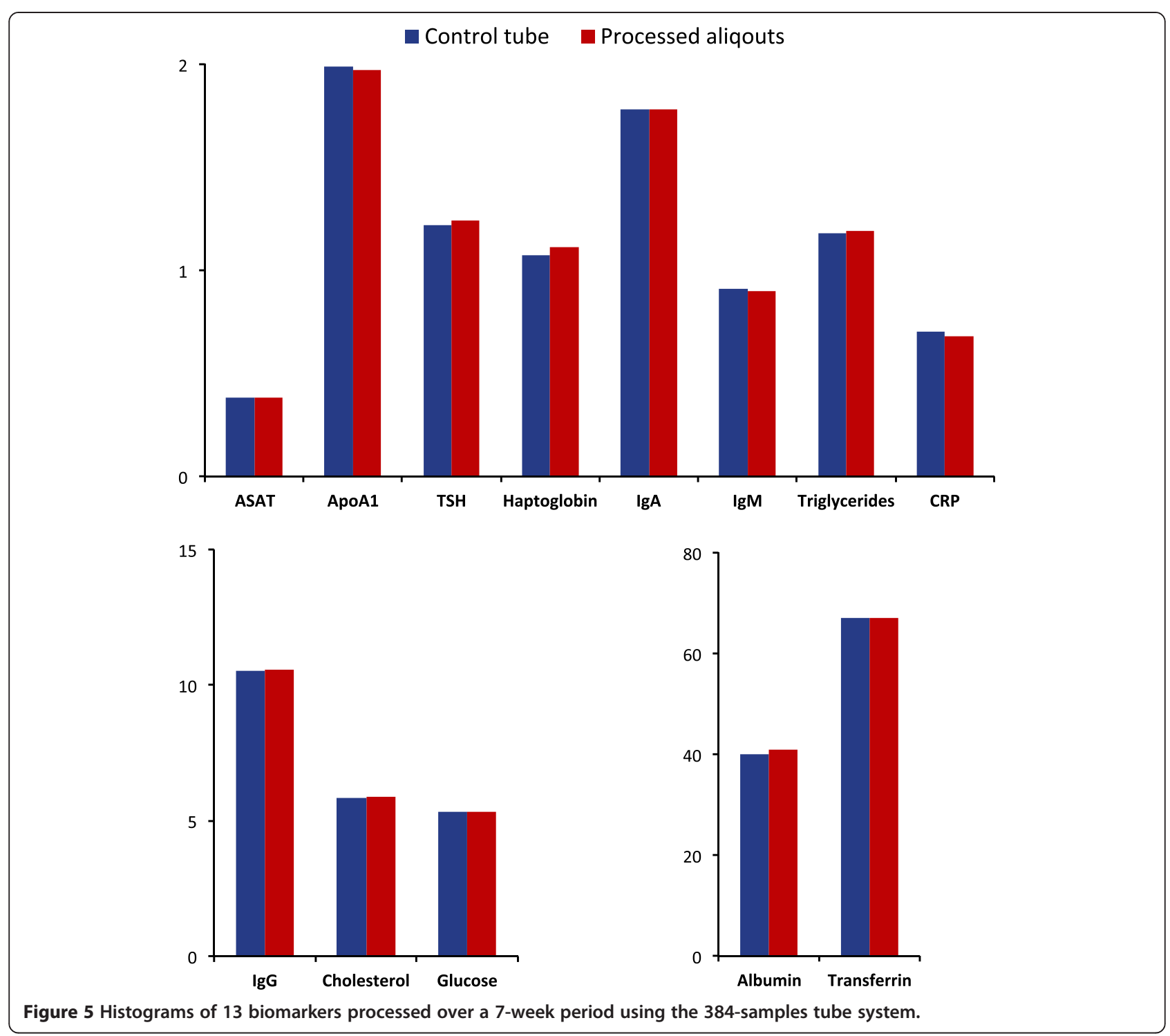

systems. New methods for providing conditions of stable storage are currently being developed. Automatic robotic systems will be an essential component for the success of standardization procedures that speak to the preservation of sample integrity. This will be especially needed in studies of large number patient cohorts where samples are collected and processed in multitudes of laboratories at national and global levels of study. High cost that is associated with investments in robotic instrumentation is a challenge in a high quality driven healthcare society. Large scale processing on the other hand puts extremely high demands on manual sample handling and is close to impossible. As ultra-low temperatures demands for increased power consumptions, the lower temperatures also brings another challenge along that might be a disadvantage and that is huge temperature alterations upon power cuts or other electronic problems that ultimately will change the temperature of the sample.

In addition to their usefulness in clinical decision making, stable biobanked samples are also of importance to drug development, biomarker identification, sentinel studies of health and disease in society in both academic and regulatory settings [24].

\section{Competing interests}

Paul Upton and Stephanie Carter are employees of Thermo Fisher Scientific Magnus Dahlbäck is an employee of AstraZeneca R\&D. There is no financial gain to the companies expected through publication of this manuscript. The remaining authors have no competing interests related to this manuscript.

Authors' contributions

Conception and design: GMV, TEF, HL, JM. Analysis and interpretation: PD, $H L, A V, C W, K S, E W, M R$. Drafting the manuscript for important intellectual content: GMV, JM, PU, SC, RA, MD, DE. All authors read and approved the final manuscript. 


\section{Acknowledgements}

This work was supported by grants from the Swedish Academy of Pharmaceutical Sciences, C-HPP (HUPO), Swedish Research Council, the Swedish Foundation for Strategic Research (SSF, TOTAL AMI), Vinnova, Ingabritt \& Arne Lundbergs forskningsstiftelse, Fundacion Federico SA, and by the Crafoord Foundation.

\section{Author details}

${ }^{1}$ Department of Laboratory Medicine, Section for Clinical Chemistry, Lund University, Skåne University Hospital in Malmö, Malmö 205 02, Sweden.

${ }^{2}$ Clinical Protein Science \& Imaging, Biomedical Center, Dept. of Measurement Technology and Industrial Electrical Engineering, Lund University, BMC C13, 22184 Lund, Sweden. ${ }^{3}$ Region Skåne R\&D Center, Region Skåne, 22185 Lund, Sweden. ${ }^{4}$ Department of Oncology, Clinical Sciences, Lund University, 22185 Lund, Sweden. ${ }^{5}$ Region Skåne Biobank, Skåne University Hospital, 22185 Lund, Sweden. ${ }^{6}$ Thermo Fisher Scientific, Stafford House, 1 Boundary Park, Hemel Hempstead, Hertfordshire HP2 7GE, UK. ${ }^{7}$ Thermo Fisher Scientific, Rochester, MA 14625, USA. ${ }^{8}$ Respiratory and Inflammation Therapy Area, Astra Zeneca R\&D, 43183 Mölndal, Sweden. ${ }^{9}$ Department of Cardiology, Lund University, Skåne University Hospital, 221 85 Lund, Sweden. ${ }^{10}$ First Department of Surgery, Tokyo Medical University, 6-7-1 Nishishinjiku Shinjiku-ku, Tokyo 160-0023 Japan.

Received: 19 June 2013 Accepted: 7 August 2013 Published: 30 August 2013

\section{References}

1. Blow N: Biobanking: freezer burn. Nat Meth 2009, 6:173-178.

2. Marko-Varga G, Végvári Á, Welinder C, Lindberg H, Rezeli M, Edula G, Svensson KJ, Belting M, Laurell T, Fehniger TE: Standardization and utilization of biobank resources in clinical protein science with examples of emerging applications. J Proteome Res 2012, 11:5124-5134.

3. Vaught J, Rogers J, Carolin T, Compton C: Biobankonomics: developing a sustainable business model approach for the formation of a human tissue biobank. JNCI Monogr 2011, 2011:24-31.

4. Hewitt RE: Biobanking: the foundation of personalized medicine. Current opin oncol 2011, 23:112-119.

5. Eiseman E, Bloom G, Brower J, Clancy N, Olmsted SS: Case Studies of Existing Human Tissue Repositories: "Best Practices" for a Biospecimen Resource for the Genomic and Proteomic Era. Santa Monica, CA: RAND Corporation; 2003.

6. Hallmans G, Vaught JB: Best practices for establishing a biobank. In Methods in Biobanking, Volume 675. Edited by Dillner J. Totowa: Humana Press Inc; 2011:241-260.

7. Vaught J, Rogers J, Myers K, Lim MD, Lockhart N, Moore H, Sawyer S, Furman $J$, Compton C: An NCl perspective on creating sustainable biospecimen resources. JNCI Monogr 2011, 2011:1-7.

8. Moore HM, Kelly AB, Jewell SD, McShane LM, Clark DP, Greenspan R, Hayes DF, Hainaut $P$, Kim $P$, Mansfield $E$, et al: Biospecimen Reporting for Improved Study Quality (BRISQ). J Proteome Res 2011, 10:3429-3438.

9. Herbert G, George G, Johannes S: Connecting the public with biobank research: reciprocity matters. Nat Rev Genet 2011, 12:738-739.

10. Nietfeld JJ, Sugarman J, Litton J-E: The Bio-PIN: a concept to improve biobanking. Nat Rev Cancer 2011, 11:303-308.

11. Hamburg MA, Collins FS: The path to personalized medicine. New Engl J Med 2010, 363:301-304.

12. Fullerton SM, Anderson NR, Guzauskas G, Freeman D, Fryer-Edwards K: Meeting the governance challenges of next-generation biorepository research. Sci Transl Med 2010, 2:15 cm 13.

13. Shaw PM, Patterson SD: The value of banked samples for Oncology Drug Discovery and Development. JNCI Monogr 2011, 2011:46-49.

14. Marko-Varga G: BioBanking - The Holy Grail of novel drug and diagnostic developments? J Clin Bioinform 2011, 1:14.

15. Hallmans $G$, Vaught JB: Best practices for establishing a biobank. Methods Mol Biol 2011, 675:241-260.

16. Vaught J, Rogers J, Myers K, Lim MD, Lockhart N, Moore H, Sawyer S Furman $J$, Compton C: An NCl perspective on creating sustainable biospecimen resources. J Nat/ Cancer Inst Monogr 2011, 2011:1-7.

17. Malm J, Végvári Á, Rezeli M, Upton P, Danmyr P, Nilsson R, Steinfelder E, Marko-Varga G: Large scale biobanking of blood - The importance of high density sample processing procedures. J Proteomics 2012, 76:116-124.
18. Sepper R, Ross P, Tiik M: Nationwide health data management system: a novel approach for integrating biomarker measurements with comprehensive health records in large populations studies. $J$ Proteome Res 2010, 10:97-100.

19. LaBaer J: Improving International Research with clinical specimens: 5 achievable objectives. J Proteome Res 2012, 11:5592-5601.

20. Marko Varga GA, Végvári Á, Fehniger TE: A protein shake-up. Public Serv Rev Eur Union 2011, 21:250-252. 250-252.

21. Zatloukal K, Hainaut P: Human tissue biobanks as instruments for drug discovery and development: impact on personalized medicine. Biomark Med 2010, 4:895-903.

22. Knoppers BM, Isasi R: Stem cell banking: between traceability and identifiability. Genome Med 2010, 2:73.

23. Anderlik M: Commercial biobanks and genetic research: ethical and legal issues. Am J Pharmacogenomics 2003, 3:203-215.

24. Végvári Á, Welinder C, Lindberg H, Fehniger TE, Marko-Varga G: Biobank resources for future patient care: developments, principles and concepts. J Clin Bioinform 2011, 1:24.

doi:10.1186/2213-0802-1-14

Cite this article as: Malm et al:: Standardization developments for large scale biobanks in smoking related diseases - a model system for blood sample processing and storage. Translational Respiratory Medicine 2013 1:14.

\section{Submit your manuscript to a SpringerOpen ${ }^{\odot}$ journal and benefit from:}

- Convenient online submission

- Rigorous peer review

- Immediate publication on acceptance

- Open access: articles freely available online

- High visibility within the field

- Retaining the copyright to your article

Submit your next manuscript at $>$ springeropen.com 